\title{
Clinical Presentation of Celiac Disease Masks Therapeutic Perspectives of Celiac Disease
}

\author{
Sur G Genel ${ }^{1,2 *}$, Floca D Emanuela1, Sur M Lucia ${ }^{1,3}$, Sur G Daniel ${ }^{1}$ and Samasca Gabriel ${ }^{1,2}$
}

${ }^{1}$ University of Medicine and Pharmacy, Iuliu Hatieganu, Cluj-Napoca, Romania

${ }^{2}$ Emergency Clinical Hospital for Children, Cluj-Napoca, Romania

${ }^{3}$ Department of Rheumatology, Iuliu Hatieganu, Cluj-Napoca, Romania

\begin{abstract}
Celiac disease is a clinical entity that is not difficult to diagnose when it shows the classic symptoms. It was found that there are other forms of celiac disease that are difficult to diagnose because of atypical manifestations. The aim of this study is to describe the prevalence of different forms of celiac disease in accordance with 'The Oslo definitions for celiac disease and related terms' and therapeutic perspectives of this disease.
\end{abstract}

Keywords: Celiac disease; Transglutaminase antibodies; Intestinal mucosa

\section{Background}

Celiac disease $(\mathrm{CD})$ is a gluten-sensitive, immune-mediated chronic enteropathy with a wide range of manifestations of variable severity. This disease is due to the damage of intestinal mucosa as a result of the action of gluten, in genetic predisposed people. The masks that can diagnose celiac disease, presented below, are divided into: classical CD, non-classical CD, subclinical CD and potential CD. The clinical manifestations and histology changes of small bowel mucosa will disappear with gluten-free diet, but histological relapses will appear in a variable period of time after re-use of foods with gluten. Prevalence of this disorder is difficult to estimate due to the variability of clinical presentation. Lifelong avoidance of gluten ingestion is the main treatment for patients with celiac disease [1-3].

In two years (january 2010-december 2012), there were 144 cases of celiac disease identified among the patients of the Clinical Emergency Pediatric Hospital Cluj-Napoca, Clinic of Pediatrics II, by determining IgA anti-tissue transglutaminase antibodies. The screening involved 1280 children who had suggestive signs of celiac disease (chronic diarrhea, weight stagnation or weight loss). We obtained parental consent for children to be included in the study in accordance with the confidentiality norms.

\section{Clinical Presentation}

According to The Oslo definitions for celiac disease [1] there are 4 possible presentations of celiac disease.

\section{Classical celiac disease}

It is characterized by gastrointestinal signs and symptoms. Typical form of the disease begins at various times after foods that contain gluten are introduced into the diet. Characteristic manifestations of infants and young children are chronic diarrhea, vomiting, anorexia, abdominal distention, abdominal pain, and poor weight gain or weight loss. Nutritional deficiency syndrome occurs in time and its manifestations are: growth deficiency, psychomotor development deficiency, anemia, rickets, and hemorrhagic manifestations. Undiagnosed and severely affected infants may present celiac crisis characterized by explosive diarrhea, marked abdominal distention, dehydration and lethargy. Delayed diagnosis and persistent symptoms can cause severe malnutrition [1,2].
The onset of celiac disease with gastrointestinal symptoms in older children may occur at any age. These patients may present nausea, loss of appetite, abdominal discomfort, constipation, and intermittent diarrhea. It is important to note that the patient's age at the onset of symptoms may be influenced by the amount of gluten in the diet and the duration of breast feeding $[1,2,4]$.

\section{Non-classical celiac disease}

It is estimated that approximately $70 \%$ of newly diagnosed celiac disease patients do not present typical gastrointestinal manifestations. Onset of illness in infants and toddlers include gastrointestinal symptoms and failure to thrive. Minor gastrointestinal symptoms, height and weight growth deficiency, delayed puberty predominates in clinical presentation of celiac disease in childhood-onset. Anemia is the most common manifestation in teenagers and young adults $[1,2,4]$.

Celiac disease includes extraintestinal manifestations. Dermatitis herpetiformis is noticed as a manifestation in adolescents and adults. It is characterized by skin blisters and rashes, lesions that are present on the elbows, knees and buttocks. Dental enamel hypoplasia affects only permanent dentition and may be the only manifestation of celiac disease. Iron-deficiency anemia that is resistant to oral iron therapy often completes clinical presentation of the disease. Children and adolescents often present short stature and constitutional delay of puberty. Adolescent girls with untreated celiac disease may present delayed onset of menarche. It is estimated that about half of the children with celiac disease have a low bone mineral density at the time of diagnosis; bone damage can reach osteoporosis. Neurologic and psychiatric disorders including depression, anxiety, irritability, autism, peripheral neuropathy, cerebellar ataxia, epilepsy and migraines have all been reported. Patients with non-classical form of celiac disease can present reproductive diseases, arthritis and arthralgia and liver

*Corresponding author: Sur G Genel, University of Medicine and Pharmacy, luliu Hatieganu, Cluj-Napoca, Romania, E-mail: surgenel@yahoo.com

Received April 01, 2013; Accepted April 20, 2013; Published April 25, 2013

Citation: Genel SG, Emanuela FD, Lucia SM, Daniel SG, Gabriel S (2013) Clinical Presentation of Celiac Disease Masks Therapeutic Perspectives of Celiac Disease. Pharmaceut Anal Acta 4: 228. doi:10.4172/2153-2435.1000228

Copyright: $\odot 2013$ Genel SG, et al. This is an open-access article distributed under the terms of the Creative Commons Attribution License, which permits unrestricted use, distribution, and reproduction in any medium, provided the original author and source are credited. 
dysfunction. These conditions usually respond to a gluten-free diet [13].

Celiac disease has been associated with many other autoimmune conditions and genetic syndromes. Associated genetic syndromes include Down syndrome, Williams syndrome, and Turner syndrome. The most common autoimmune diseases associated with celiac disease are the Type 1 diabetes mellitus and thyroiditis $[4,5]$.

\section{Subclinical celiac disease}

In an apparently healthy child, the analytical findings and the biopsy are consistent with a diagnosis of celiac disease. Many of these children belong to risk groups and suffer from other autoimmune conditions (diabetes type I, thyroiditis, hepatitis, psoriasis, etc.), genetic conditions (Down's syndrome, Turner's syndrome, Williams syndrome, IgA deficiency) or are first degree relatives to patients with such conditions [1]

\section{Potential celiac disease}

This refers to children with positive, analytical evidence, but normal intestinal biopsies or only minimally abnormal changes $[1,5]$.

In terms of manifestation of celiac disease masks, the 144 patients are divided as follows: 66 cases with classical CD, 69 cases with nonclassical CD, 6 cases with subclinical CD and 3 cases with potential CD (Table 1).

In our study classical and non-classical forms of celiac disease are almost equally represented. Classical form has symptoms that allow early diagnosis while non-classical form involves problems of differential diagnosis. Patients with subclinical form of celiac disease are apparently healthy, while diagnostic tests are positive. Potential form is opposite compared to subclinical form, in which patients have symptoms but diagnostic tests are negative. Refractory form is the fifth manifestation of celiac disease. It is characterized by persistent or recurrent signs and symptoms of malabsorption with villous atrophy despite the gluten-free diet. This form was not found in our cases.

\section{Treatment}

A gluten free diet is the only available treatment for celiac disease. Wheat, barley and rye are grains that have to be avoided in the diet. Avoiding gluten in the diet has good effects on symptoms. Compliant patients improve their weight and height; children regain their lost psychomotor assets; behavioral disorders disappear; hematological and biochemical parameters normalize [6,7].

There are some alternative future celiac disease treatments strategies. These include the following: hydrolysis of toxic gliadin peptide, prevention of toxic gliadin peptide absorption, blockage of deamidation of specific glutamine residues by tissue, restoration of immune tolerance towards gluten, modulation of immune response to dietary gliadin, and restoration of intestinal architecture [6].

\begin{tabular}{|c|c|c|c|}
\hline $\begin{array}{c}\text { Clinical } \\
\text { presentation }\end{array}$ & $\begin{array}{c}\text { Number of } \\
\text { cases }\end{array}$ & Percentage & Comments \\
\hline Classical CD & 66 & $45.83 \%$ & form of CD diagnosed most easily \\
\hline Non-classical CD & 69 & $47.92 \%$ & the most frequent form of CD \\
\hline Subclinical CD & 6 & $4.17 \%$ & involve diagnostic problems \\
\hline Potential CD & 3 & $2.08 \%$ & difficult to sustain \\
\hline & 144 & $100 \%$ & \\
\hline
\end{tabular}

Table 1: Distribution of clinical forms of celiac disease cases included in the study.

\section{Hydrolysis of toxic gliadin peptide}

In patients with celiac disease gluten peptides are resistant to digestion by intestinal proteases. Enzyme supplementation therapy with prolyl endopeptidase, and glutenenases and endoprotease have been proposed to splinter peptides involved in the pathogenesis of the disease, thus abolishing the immune response. An alternative approach involves pretreatment of gluten-containing foods with bacterialderived peptidases (Lactobacilli) responsible for lyse the proline-/ glutamine-rich gluten peptides, also reducing the immune response $[3,6]$.

\section{Prevention of toxic gliadin peptide absortion}

Larazotide, a synthetic hexapeptide derived from the Zonula Occludens toxin of Vibrio Cholera, inhibits the opening of tight junctions of the small intestine epithelial cells. Gliadin can be absorbed both paracellular route and transcellular pathways, so this therapeutic modality should be combined with another. Synthetic polymer poly and egg yolk anti-gliadin antibody can prevent absorption gliadin. Their effects in patients with celiac disease are still studying [6].

Blockage of selective deamidation of specific glutamine residues by tissue transglutaminase 2 inhibitor

It could be a way to inhibit the inflammatory process upon gluten ingestion.

\section{Restore immune tolerance towards gluten}

There is clinical trials studying the effects of vaccine application. Thus peptide vaccine containing a mixture of immunotoxic $\alpha$ - and $\omega$ gliadins and B-hordein, and dermal inoculation of human hookworm could be used to modulate the immune response to gluten [8].

\section{Modulation of immune response to dietary gliadin}

Considering the pathogenesis of celiac disease modulating the immune response is attempted by the discovery of HLA-DQ blocker and an interleukin blocker $[3,6]$.

\section{Restoration of intestinal architecture by R-spondin-1}

It has not yet been tested on humans to be considered as a therapeutic option for celiac disease [6]. In our study treatment for all clinical forms of celiac disease was gluten-free diet. According to the recommendations patients received iron, vitamin of group B, Vitamin A, D, E, K, calcium

\section{References}

1. Husby S, Koletzko S, Korponay-Szabó IR, Mearin ML, Phillips A et al. (2012) European Society for Pediatric Gastroenterology, Hepatology, and Nutrition guidelines for the diagnosis of coeliac disease. J Pediatr Gastroenterol Nutr 54: 136-160.

2. Reilly NR, Fasano A, Green PH (2012) Presentation of celiac disease Gastrointest Endosc Clin N Am 22: 613-621.

3. Armstrong, Hegade VS, Robins G (2012) Advances in celiac disease. Curr Opin Gastroenterol 28: 104-112.

4. Tanpowpong P, Broder-Fingert S, Katz AJ, Camargo CA Jr (2012) Age-related patterns in clinical presentations and gluten-related issues among children and adolescents with celiac disease. Clin Transl Gastroenterol 3: e9.

5. Mearin ML (2007) Celiac disease in children and adolescents. Curr Prob Pediatr Adolesc Health Care 37: 86-105.

6. Gujral N, Freeman HJ, ThomsonAB (2012) Celiac disease: Prevalence, diagnosis, pathogenesis and treatment. World J Gastroenterol 18: 6036-6059.

7. Troncone R, Ivarsson A, Szajweska H, Mearin ML, Members of European 
Citation: Genel SG, Emanuela FD, Lucia SM, Daniel SG, Gabriel S (2013) Clinical Presentation of Celiac Disease Masks Therapeutic Perspectives of Celiac Disease. Pharmaceut Anal Acta 4: 228. doi:10.4172/2153-2435.1000228

Page 3 of 3

Multistakeholder Platform on CD (CDEUSSA) (2008) Future research on celiac disease - a position report from the European multi-stake-holder platform on celiac disease (CDEUSSA). Aliment Pharmacol Ther 27: 1030-1043.
8. Daveson AJ, Jones DM, Gaze S, McSorley H, Clouston A, et al. (2011) Effect of hookworm infection on wheat challenge in celiac disease--a randomised double-blinded placebo controlled trial. PLoS One 6: e17366. 